\title{
Metabolite Ratio for AUC Infinity Predicted
}

National Cancer Institute

\section{Source}

National Cancer Institute. Metabolite Ratio for AUC Infinity Predicted. NCI Thesaurus.

Code C156566.

The ratio of AUC infinity predicted for the metabolite to the AUC infinity predicted for the parent analyte. 